INTERVENTIONAL CARDIOLOGY AND SURGERY

\title{
Serial six year quantitative angiographic follow up in asymptomatic patients following successful coronary angioplasty
}

\author{
Y Takeda, T Kobayashi, N Awata, S Sato, J H C Reiber, T Nakagawa, E Tsuchikane, O Katoh, \\ M Kirino, T Kobayashi, K Yachiku, N Shibata
}

Heart 2004;90:1179-1182. doi: 10.1136/hrt.2003.022772

\begin{abstract}
See end of article for authors' affiliations

Correspondence to: Dr Y Takeda, Department of Cardiology, Osaka Medical Centre for Cancer and Cardiovascular Diseases, 1-3-3 Nakamichi, Higashinariku, Osaka City, Osaka 5378511, Japan; takeda@ xk9.so-net.ne.jp
\end{abstract}

Accepted 26 January 2004

\begin{abstract}
Objective: To evaluate long term (six years) lumen changes after balloon angioplasty by using quantitative coronary angiography.

Methods: Complete serial quantitative coronary angiography (before and after angioplasty and at six months, three years, and six years) was performed in 100 patients with successful angioplasty and without subsequent repeated revascularisation. In all, 198 dilated segments were compared with 395 non-dilated segments that were obtained from non-target arteries of study patients.

Results: From six months to three years affer angioplasty, minimum lumen diameter (MLD) increased significantly by $0.13(0.28)$ (mean (SD)) $\mathrm{mm}$ in dilated segments and decreased significantly by 0.04 $(0.27) \mathrm{mm}$ in non-dilated segments. From three years to six years, MLD remained stable in dilated segments but decreased further (by $0.04(0.28) \mathrm{mm}$ ) in non-dilated segments. Consequently, the $\triangle M L D$ between six months and six years was larger in dilated segments than in non-dilated segments $(0.12$ $(0.32) v-0.08(0.34) ; p<0.001)$. Further, $\Delta M L D$ from six months to six years correlated positively with the percentage diameter stenosis (DS) at six months in each group (dilated segments $r=0.47$, $p<0.0001$; non-dilated segments $r=0.49, p<0.0001$ ). Multivariate analysis showed that the only independent predictor of $\triangle M L D$ over six years for each group was the DS at six months.

Conclusions: Lesion regression occurs within the first three years after angioplasty and reaches a plateau thereafter. Moreover, the stenosis severity at six months predicts the magnitude of late regression affer angioplasty.
\end{abstract}

$\mathrm{S}$ tenting is now the predominant type of catheter based coronary intervention. In a recent study of long term quantitative coronary angiography (QCA) follow up after stenting, Kimura and colleagues ${ }^{1}$ showed that late ( $>4$ years) luminal renarrowing was common. Whether this late renarrowing phenomenon is specific to stenting is unclear because few studies have provided long term $(\geqslant 5$ years) QCA information after other interventional procedures, including balloon angioplasty. ${ }^{23}$

The aim of the present study was to investigate changes in lesion regression over a six year period after angioplasty and to determine which clinical and angiographic factors are associated with these changes.

\section{METHODS}

\section{Study population}

PCS (prevention of coronary sclerosis) was a prospective quantitative angiographic study with three serial (baseline, two year, and five year follow up) angiograms designed to test the effect of five years of pravastatin treatment on the progression of coronary atherosclerosis in normocholesterolaemic patients with coronary artery disease. The results of PCS have been published. ${ }^{4}$

A total of 100 patients from the 329 patients of the original PCS cohort were enrolled into the present study according to the following criteria: (1) successful balloon angioplasty to de novo native coronary artery disease within one year before entry into PCS; (2) surviving clinically stable patients who did not undergo subsequent repeated revascularisation; (3) QCA data measured serially before and after angioplasty, at baseline, and at two year and five year follow up time points as part of the original PCS; and (4) the presence of nondilated site(s) obtained from non-target arteries during the initial procedure. Because the angioplasty technique itself may have accelerated disease proximal or distal to the dilated lesion as a result of endothelial injury, non-dilated segments in the treated arteries were excluded from analysis. Thus, 83 patients who underwent angioplasty to native three vessel disease during the initial procedure were excluded.

Exclusion criteria were: (1) death during follow up (11 patients); (2) catheter based interventions for new lesions that were unrelated to any dilated segments during the initial procedure (20 patients); (3) no follow up angiography (17 patients); (4) non-qualifying angiography for QCA analysis (24 patients); and (5) absence of segments treated with angioplasty (74 patients). These criteria accounted for the 146 patients of the original PCS cohort who were excluded from the present study.

The study protocol was approved by our institutional ethics committee. All patients gave written informed consent.

\section{Balloon angioplasty procedure and QCA}

Coronary angiography and angioplasty were performed according to standard techniques. ${ }^{4}$ Successful angioplasty was defined as the absence of major ischaemic complications, $<50 \%$ residual stenosis, and $>30 \%$ residual stenosis increase by visual estimation. Follow up angiography was performed at 6.8 (2.5) months, 36.2 (4.5) months, and 71.8 (4.7) months after the initial procedure, which is roughly

Abbreviations: DS, percentage diameter stenosis; MLD, minimum lumen diameter; PCS, prevention of coronary sclerosis; QCA, quantitative coronary angiography 
equivalent to and designated as six months, three years, and six years, respectively. Coronary angiograms were submitted to the Heart Core in Leiden, the Netherlands, and analysed quantitatively in a blinded manner with the Cardiovascular Measurement System (CMS-MEDIS Medical Imaging Systems, Leiden, the Netherlands). For QCA, the coronary artery tree was divided into 15 segments on the basis of the American Heart Association classification. ${ }^{5}$ For all QCA evaluable segments, reference diameter, minimum lumen diameter (MLD), and percentage diameter stenosis (DS) were analysed as previously described. ${ }^{4}{ }^{6}$

\section{Statistical analysis}

Data are presented as mean (SD) or as frequencies. Repeated measures analysis of variance was used to establish any significant differences over time in reference diameter, MLD, and DS for the dilated and non-dilated segments. Withingroup data (after repeated measures analysis of variance) were compared with the paired $t$ test. Between-group data were compared with the unpaired $t$ test. All $t$ tests were adjusted with the Bonferroni correction for multiple comparisons. A probability value of $\mathrm{p}<0.05$ was considered significant. Categorical variables were compared with the $\chi^{2}$ test or Fisher's exact test.

To identify associations between $\triangle$ MLD from six months to six years and clinical or angiographic factors including pravastatin usage, conventional risk factors, and QCA measurements, linear regression analyses were performed. Predictors were determined by stepwise linear regression with an entry criterion of 0.20 and a stay criterion of 0.05 .

\section{RESULTS}

Baseline clinical and angiographic characteristics

Table I summarises the baseline characteristics in the study cohort of 100 patients who had complete serial angiograms and the 17 patients who did not have either a three or a six year follow up angiogram. No significant differences were observed between the two groups. In total, 198 dilated and 395 non-dilated segments were characterised from 100 study patients.

Table 1 Baseline characteristics in the study cohort of 100 patients who had complete serial angiograms and 17 patients who did not have either three or six year follow up angiography

\begin{tabular}{lll}
\hline & Complete series & No follow up \\
\hline Patient characteristics & 100 & \\
Number of patients & $81(81 \%)$ & 17 \\
Men & $58.6(38-69)$ & $16(94 \%)$ \\
Age (years) & $17(17 \%)$ & $59.1(39-69)$ \\
Extent of coronary artery diseases & $83(83 \%)$ & $3(18 \%)$ \\
1 vessel disease & $56(56 \%)$ & $14(82 \%)$ \\
2 vessel disease & $18(18 \%)$ & $11(65 \%)$ \\
Previous myocardial infarction & $50(50 \%)$ & $4(24 \%)$ \\
Diabetes mellitus & $53(53 \%)$ & $10(59 \%)$ \\
Hypertension & $54(54 \%)$ & $10(59 \%)$ \\
Hypercholesterolaemia & & $11(65 \%)$ \\
Smoking & $93(93 \%)$ & \\
Medication & $71(71 \%)$ & $15(88 \%)$ \\
Long acting nitrate & $27(27 \%)$ & $10(59 \%)$ \\
Calcium channel blocker & $68(68 \%)$ & $4(24 \%)$ \\
$\beta$ Blocker & $5.88(1.36)$ & $14(82 \%)$ \\
Pravastatin & $3.88(1.29)$ & $6.15(1.83)$ \\
Total cholesterol (mmol/I) & $1.23(0.32)$ & $4.50(1.90)$ \\
Low density lipoproteins (mmol/I) & $1.69(1.06)$ & $1.06(0.26)$ \\
High density lipoproteins (mmol/I) & 198 & $1.94(1.58)$ \\
Triglycerides (mmol/l) & & 28 \\
Angiographic and procedural characteristics & & \\
Number of lesions & $99(50 \%)$ & $11(39 \%)$ \\
Lesion location & $52(26 \%)$ & $9(32 \%)$ \\
LAD & $46(23 \%)$ & $8(29 \%)$ \\
Right & $1(1 \%)$ & $0(0 \%)$ \\
LCx & $79 / 119(40 \% / 60 \%)$ & $16 / 12(57 \% / 43 \%)$ \\
Left main & $6(3 \%)$ & $1(4 \%)$ \\
AHA/ACC type & $13.9(7.4)$ & $13.6(3.2)$ \\
B1/B2C & $2.62(0.75)$ & $2.74(0.54)$ \\
Calcified & $0.76(0.54)$ & $0.68(0.47)$ \\
Before angioplasty & $71.0(18.5)$ & $2.82(15.3)$ \\
Lesion length (mm) & $2.67(0.71)$ & $2.14(0.62)$ \\
RD (mm) & $2.00(0.68)$ & $24.0(13.0)$ \\
MLD (mm) & $24.9(15.9)$ & $3.0(0.5)$ \\
DS (\%) & $2.9(0.4)$ & $1.1(0.2)$ \\
Immediately after angioplasty & $1.1(0.3)$ & $8.3(2.4)$ \\
RD (mm) & $7.3(2.6)$ & $157(16))$ \\
MLD (mm) & $131(81)$ & \\
DS (\%) & $30(15 \%)$ & \\
Balloon diameter (mm) & & \\
Balloon to artery ratio & & \\
Inflation pressure (atm) & & \\
Inflation time (s) & & \\
Dissection & & \\
\hline & & \\
\hline
\end{tabular}

Data are mean (SD), mean (range), or number (\%).

AHA, American Heart Association; ACC, American College of Cardiology; DS, percentage diameter stenosis; $L A D$, left anterior descending; $L C x$, left circumflex; MLD, minimum lumen diameter; RD, reference diameter. 
Table 2 Serial quantitative angiographic results at six months, three years, and six years for dilated and non-dilated segments

\begin{tabular}{|c|c|c|}
\hline Variable & Dilated segments $(n=198$ ) & Non-dilated segments $(n=395$ ) \\
\hline \multicolumn{3}{|l|}{6 months' follow up } \\
\hline $\mathrm{RD}(\mathrm{mm})$ & $2.52(0.63)$ & $2.84(0.84)$ \\
\hline$M L D(m m)$ & $1.72(0.51)$ & $2.14(0.72)$ \\
\hline DS $(\%)$ & $31.9(12.0)$ & $24.7(11.0)$ \\
\hline \multicolumn{3}{|l|}{3 years' follow up } \\
\hline $\mathrm{RD}(\mathrm{mm})$ & $2.55(0.61)$ & $2.81(0.81)$ \\
\hline $\mathrm{MLD}(\mathrm{mm})$ & $1.84(0.48)$ & $2.10(0.67)$ \\
\hline DS (\%) & $27.4(11.1)$ & $25.4(9.8)$ \\
\hline \multicolumn{3}{|l|}{6 years' follow up } \\
\hline $\mathrm{RD}(\mathrm{mm})$ & $2.55(0.61)$ & $2.80(0.84)$ \\
\hline $\mathrm{MLD}(\mathrm{mm})$ & $1.83(0.46)$ & $2.06(0.68)$ \\
\hline DS $(\%)$ & $27.4(10.9)$ & $26.4(10.4)$ \\
\hline \multicolumn{3}{|l|}{ Change in MLD (mm) } \\
\hline From 6 months to 3 years & $0.13(0.28)^{*} \dagger$ & $-0.04(0.27)^{*}$ \\
\hline From 3 years to 6 years & $-0.01(0.24)$ & $-0.04(0.28)^{*}$ \\
\hline From 6 months to 6 years & $0.12(0.32)^{*} \dagger$ & $-0.08(0.34)^{*}$ \\
\hline \multicolumn{3}{|l|}{ Change in DS (\%) } \\
\hline From 6 months to 3 years & $-4.49(10.4)^{*} \dagger$ & $0.65(9.2)$ \\
\hline From 3 years to 6 years & $-0.01(9.1)$ & $1.03(9.6)^{*}$ \\
\hline From 6 months to 6 years & $-4.50(11.4)^{*} \dagger$ & $1.68(11.4)^{*}$ \\
\hline
\end{tabular}

\section{Long term QCA outcomes and predictors of $\triangle M L D$ over six years}

Table 2 summarises results of complete serial QCA at six months, three years, and six years for the dilated and nondilated segments. Between six months and three years after angioplasty, MLD increased by $0.13(0.28) \mathrm{mm}$ in the dilated segments and decreased by $0.04(0.27) \mathrm{mm}$ in the nondilated segments. From three years to six years, MLD remained stable in the dilated segments but decreased further in the non-dilated segments by $0.04 \quad(0.28) \mathrm{mm}$. Changes in DS were similar when comparing dilated and non-dilated segments. Consequently, lesion regression in dilated segments occurred within the first three years after angioplasty and there was little change in lumen dimensions beyond three years. As expected, non-dilated segments had gradual lesion progression over six years.

The $\Delta \mathrm{DS}$ from six months to six years in both groups correlated negatively with DS at six months (figs 1 and 2). Twenty two of the dilated segments had early restenosis (defined as $\geqslant 50 \%$ DS at six months) and 18 of those with early stenosis had regressed to $<50 \%$ DS at six years. No dilated segments without early restenosis developed late restenosis (defined as $\geqslant 50 \%$ DS at six years).

In addition, the $\triangle M L D$ from six months to six years in both groups correlated positively with DS at six months (dilated segments $r=0.47, \mathrm{p}<0.0001 ;$ non-dilated segments $r=0.49, \mathrm{p}<0.0001)$. By multivariate analysis, the only independent predictor of $\triangle \mathrm{MLD}$ between six months and six years for each group was DS at six months (dilated segments $p=0.0001$; non-dilated segments $p=0.0001$ ). In the nondilated segments, the relation between diabetes mellitus and $\triangle M L D$ over six years was of marginal significance $(\mathrm{p}=0.055)$.

\section{DISCUSSION}

This study showed, firstly, that lesion regression occurred within the first three years after angioplasty and reached a plateau thereafter; and secondly, that the magnitude of late lesion regression correlated significantly with increased stenosis severity at six months.

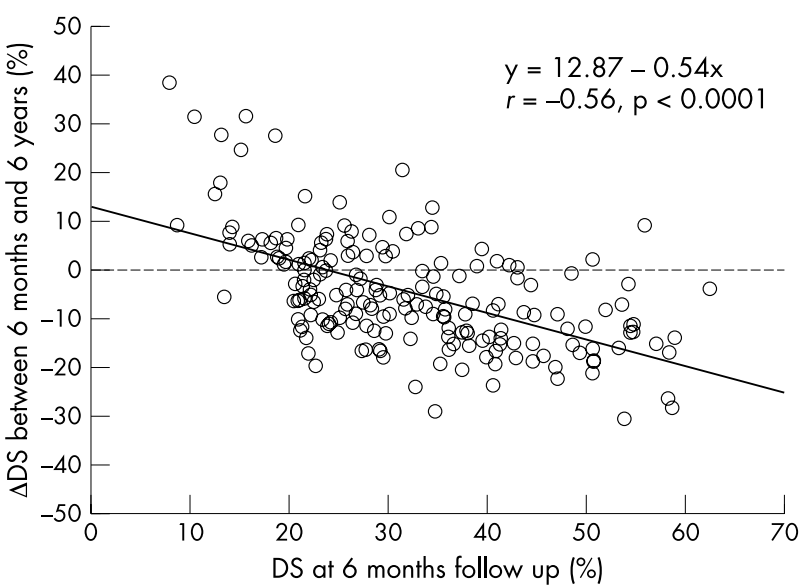

Figure 1 Change in percentage diameter stenosis ( $\triangle D S$ ) from six months to six years in the 198 dilated segments in relation to the DS at six months' follow up: $r=-0.56 ; p<0.0001$.

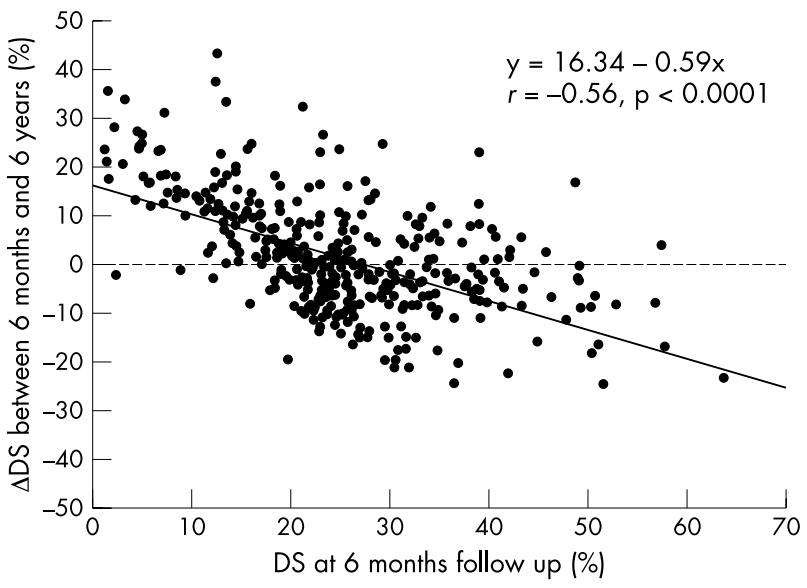

Figure $2 \Delta \mathrm{DS}$ from six months to six years in the 395 non-dilated segments in relation to the DS at six months' follow up: $r=-0.56$; $p<0.0001$. 
Previous studies of long term angiographic outcomes In a five year QCA follow up study after angioplasty, Ormiston and colleagues ${ }^{3}$ reported that lesion regression occurred at the dilated site (MLD $1.85(0.54) \mathrm{mm}$ at six months and $2.10(0.62) \mathrm{mm}$ at five years, $\mathrm{p}<0.0001)$, which was consistent with data from the present study. Furthermore, the present study showed that the late luminal response after angioplasty may be divided into two distinct phases: the first three years, characterised by luminal enlargement; and beyond three years, characterised by little change.

This late luminal response after angioplasty contrasts with the response after stenting. In a serial QCA study of 131 patients undergoing Palmaz-Schatz stenting, Kimura and colleagues $^{1}$ showed that late luminal renarrowing was common beyond four years (MLD $2.19(0.49) \mathrm{mm}$ at three years $v 1.85(0.56) \mathrm{mm}$ beyond four years, $\mathrm{p}<0.0001)$ and speculated that this phenomenon may result from the chronic inflammation that occurs around stent struts.

\section{Potential mechanisms for late regression after angioplasty}

Our finding that high grade stenosis at six months significantly affected late regression after angioplasty is consistent with previous observations, ${ }^{3}$ which indicate that late regression may be a reversal of the early restenotic process. Indeed, histological studies suggest that the size of neointimal hyperplasia reaches a plateau between $1-4$ months and gradually diminishes in size through removal of extracellular matrix. Therefore, reduction in extracellular matrix and fibrotic scar formation associated with maturation of smooth muscle cells have been implicated as possible mechanisms for late regression after angioplasty. ${ }^{1} 8$

Interestingly, the relation between the DS at six months and $\triangle M L D$ over six years was significant and positive in both non-dilated and dilated segments in the present study. This is consistent with previous observations regarding the influence of the degree of pre-existing coronary stenoses on their evolution. ${ }^{69}$ In a three year QCA follow up analysis of 348 patients with coronary artery disease, Jost and colleagues ${ }^{9}$ showed that $\triangle \mathrm{MLD}$ of the 1063 segments correlated positively with DS at baseline $(r=0.30, \mathrm{p}<0.001)$. However, this finding cannot be explained by a gradual progression of atherosclerosis secondary to diabetes, hypertension, or hypercholesterolaemia. Studies of human native coronary arteries showed that, in the presence of such systemic factors, additional localising haemodynamic factors, such as shear stress, contributes to plaque growth and vascular remodelling. ${ }^{10}{ }^{11}$ In a series of studies, Stone and colleagues ${ }^{11}$ showed that regions of low shear stress promoted plaque growth with outward remodelling, while regions of physiological shear stress remained quiescent and regions of increased shear stress exhibited outward remodelling without plaque growth. This raises the possibility that non-dilated segments with high grade stenosis, which are associated with increased local shear stress, may develop outward remodelling without plaque growth and subsequent late lumen gain. Likewise, recent studies of postangioplasty arteries showed the highly interrelated processes of vascular remodelling and shear stress. ${ }^{12}{ }^{13}$ Thus, this mechanism may account for the similarity in the relation between DS at six months and $\triangle M L D$ over six years of dilated and non-dilated segments: following the early restenotic process after angioplasty, increased shear stress may promote outward remodelling until shear stress is restored to baseline values.

\section{Study limitations}

This was a retrospective analysis. Clinical outcomes possess potential bias due to patient selection at follow up. Moreover, this study enrolled patients with both dilated and nondilated sites to characterise differences in late lumen response. In this manner, use of different segments within the same patient may influence the similarity in predictors of $\triangle M L D$ over six years when comparing the two groups. Thus, interpreting the present finding as a predictor independent of the individual patient requires some qualification.

\section{ACKNOWLEDGEMENTS}

This study was supported by a grant from Sankyo Co, Ltd, Japan.

\section{Authors' affiliations}

Y Takeda, T Kobayashi, N Awata, E Tsuchikane, O Katoh, M Kirino, T Kobayashi, K Yachiku, N Shibata, Osaka Medical Centre for Cancer and Cardiovascular Diseases, Osaka, Japan

S Sato, Osaka Medical Centre for Health Science and Promotion, Osaka, Japan

J H C Reiber, Department of Radiology, Leiden University Medical Centre, Leiden, the Netherlands

T Nakagawa, Toyonaka Municipal Hospital, Osaka, Japan

\section{REFERENCES}

1 Kimura T, Abe K, Shizuta S, et al. Long-term clinical and angiographic followup after coronary stent placement in native coronary arteries. Circulation 2002; 105:2986-91.

2 Guiteras-Val P, Varas-Lorenzo C, Garcia-Picart J, et al. Clinical and sequential angiographic follow-up six months and 10 years after successful percutaneous transluminal coronary angioplasty. Am J Cardiol 1999:83:868-74.

3 Ormiston JA, Stewart FM, Roche $\mathrm{AH}$, et al. Late regression of the dilated site after coronary angioplasty: a 5-year quantitative angiographic study. Circulation 1997;96:468-74

4 Sato S, Kobayashi T, Awata N, et al. Randomized, controlled trial of secondary prevention of coronary sclerosis in normocholesterolemic patients using pravastatin: two-year follow-up of the prevention of coronary sclerosis study. Curr Ther Res Clin Exp 2001;62:473-85.

5 Austen WG, Edwards JE, Frye RL, et al. A reporting system on patients evaluated for coronary artery disease. Report of the ad hoc committee for grading of coronary artery disease, Council on Cardiovascular Surgery, American Heart Association. Circulation 1975;51:5-40

6 Jukema JW, Bruschke AV, van Boven AJ, et al. Effects of lipid lowering by pravastatin on progression and regression of coronary artery disease in symptomatic men with normal to moderately elevated serum cholesterol levels. The regression growth evaluation statin study (REGRESS). Circulation 1995;91:2528-40.

7 Nobuyoshi M, Kimura T, Ohishi $\mathrm{H}$, et al. Restenosis after percutaneous transluminal coronary angioplasty: pathologic observations in 20 patients. J Am Coll Cardiol 1991;17:433-9.

8 Ferns GA, Avades TY. The mechanisms of coronary restenosis: insights from experimental models. Int J Exp Pathol 2000;81:63-88.

9 Jost S, Deckers JW, Nikutta P, et al. Evolution of coronary stenoses is related to baseline severity: a prospective quantitative angiographic analysis in patients with moderate coronary disease. Eur Heart J 1994; 15:648-53.

10 Glagov S. Intimal hyperplasia, vascular modeling, and the restenosis problem. Circulation 1994;89:2888-91.

11 Stone PH, Coskun AU, Kinlay S, et al. Effect of endothelial shear stress on the progression of coronary artery disease, vascular remodeling, and in-stent restenosis in humans: in vivo 6-month follow-up study. Circulation 2003; 108:438-44.

12 Thury A, van Langenhove G, Carlier SG, et al. High shear stress after successful balloon angioplasty is associated with restenosis and target lesion revascularization. Am Heart J 2002;144:136-43.

13 Wentzel JJ, Gijsen FJ, Stergiopulos N, et al. Shear stress, vascular remodeling and neointimal formation. J Biomech 2003;36:681-8. 